\title{
An Autonomous Microscopy Workflow for Structure Determination from Atomic- Resolution Images
}

Eric Schwenker ${ }^{1}$, Fatih Sen ${ }^{1}$, Colin Ophus ${ }^{2}$, Tadas Paulauskas, ${ }^{3}$ Jinglong Guo, ${ }^{3}$ Spencer Hills, ${ }^{1}$ Robert Klie, ${ }^{3}$ and Maria K. Y. Chan ${ }^{1}$

1. Center for Nanoscale Materials, Argonne National Laboratory, Lemont IL, USA

2. Molecular Foundry, Lawrence Berkeley National Laboratory, Berkeley CA, USA

3. Department of Physics, University of Illinois at Chicago, Chicago IL, USA

Knowledge of atomistic structure is essential for understanding the properties of materials. Although advances in image acquisition and detection hardware have enabled improved atomic-scale imaging, lattice imperfections (e.g. vacancies, dislocations) can degrade or obscure the contrast and resolution of direct imaging of real materials systems. While image simulation can facilitate a better understanding of the images in these instances, the typical workflows for the process of constructing atomistic models from images are manual and require expert discretion when identifying/interpreting individual features. There is a need for automated approaches to accelerate interpretation of experimental images. Such automation can be accomplished through comparisons with simulations in an iterative loop. Peak detection and image matching are fundamental operations that must be addressed in the effort to achieve such autonomy in the interpretation of atomic-resolution images.

We first develop a peak detection procedure that considers atomic-resolution images with well-resolved intensity maxima. For high angle annular dark field (HAADF) scanning transmission electron microscopy (STEM) images, these intensity maxima reveal the lateral positions of the atomic columns and, to some extent, provide composition/depth information for the structure. This information is useful for preprocessing (image scaling and alignment), or more generally, as a way to represent the imaged structure in terms of its distinctive visual features (the positions and intensities of the atomic columns). For image matching, we introduce a structure-focused approach based on the structure similarity measure (SSIM) [1] and earth movers distance (EMD) [2]. Testing is conducted on a dataset of simulated microscopy images, created using a computationally-efficient convolution method and a set of 1632 unique structure/viewing angle prototypes from the Inorganic Crystal Structure Database (ICSD), hosted by the Materials Data Facility (MDF) service. A good matching procedure will ensure that images possessing strong visual commonalities with the query are returned near to it. In Figure 1, the image matching the query is given the highest rank for all the methods tested, and in particular for the SSIM and EMD, important motifs of the structure remain intact across several of the top-ranked images. This framework not only provides an effective means for matching both pristine and distorted simulated images, but also, when the images are represented in a feature space that considers both the positions and intensities of the atomic columns, allows for the automatic identification and segmentation of structural motifs.

Integrating basic peak detection and image matching with atomistic and first-principles modeling, we show how experimental HAADF images of CdTe grain boundaries can be used in conjunction with image simulation, as a way to propose possible structures for a variety of observed material interfaces. We also suggest how to apply convolutional neural networks (CNNs) [3] in materials microscopy to develop a more robust way to not only detect peaks in an image, but also estimate both the class (atomic column, defect, background noise, etc.) and location of objects contained within atomic-resolution 
images. As a whole, this combination of image processing and computer vision tools, alongside materials modeling to evaluate energetic information, ensures that the information contained in an experimental atomic-resolution image is interpreted in an automated and physical fashion. Ultimately, such automation would enhance and accelerate materials understanding.

References:

[1] Wang et al., IEEE Trans. Image Process, 13, (2004), 600.

[2] Y. Rubner, C. Tomasi, and L. Guibas, IJCV, 40 (2000) 99.

[3] Y. LeCun, Y. Bengio, and G. Hinton, Nature, 521 (2015) 436.

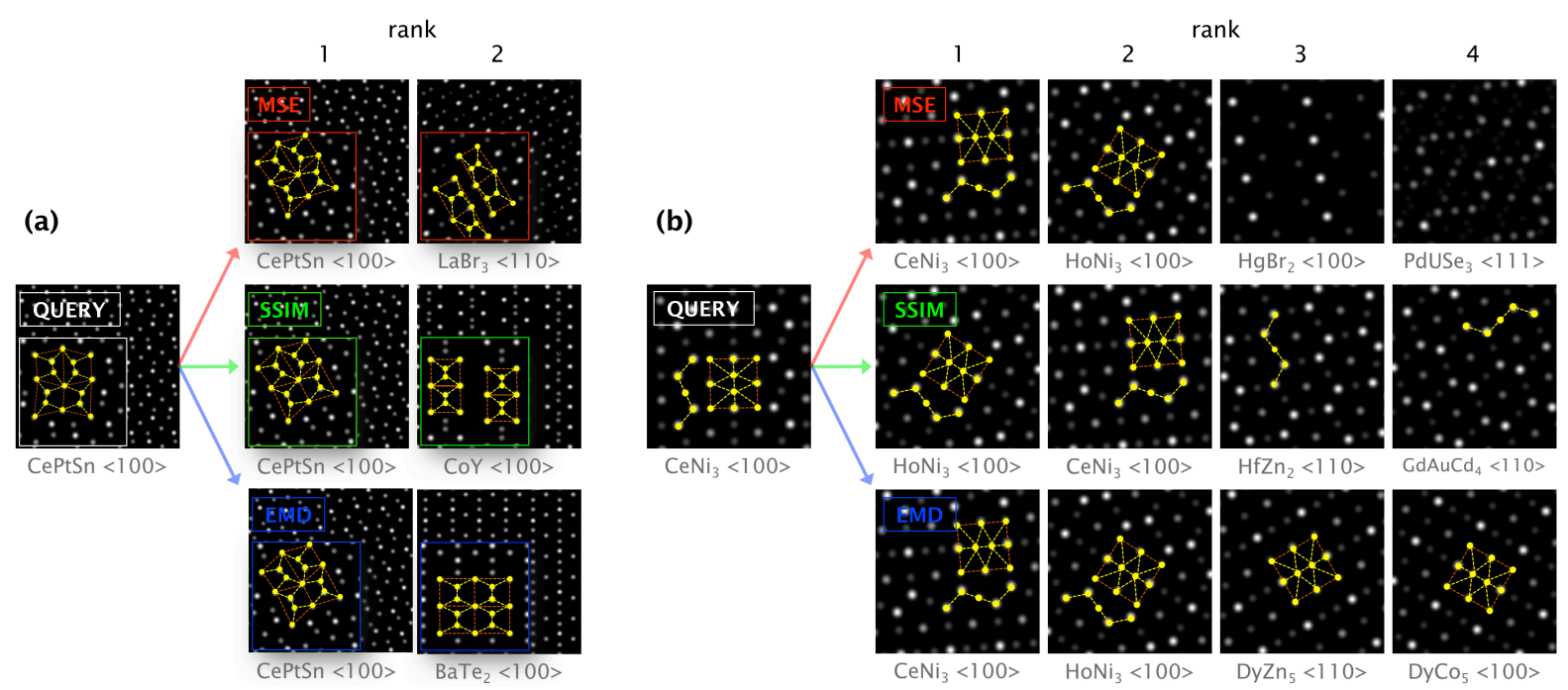

Figure 1. Comparison of the visual continuity across SSIM, and EMD top matches (compared against mean square error, MSE, as a baseline). Visual continuity is expressed as both (a) discernable local geometry similarities between a pristine query and the highest ranked matches, and the (b) extended presence of motifs from the query images, found across several matches. 